\title{
Elevated serum matrix metalloproteinase 9 (MMP-9) concentration predicts the presence of colorectal neoplasia in symptomatic patients
}

\author{
NG Hurst*, 1,2, DD Stocken', S Wilson ${ }^{3}, \mathrm{C} \mathrm{Keh}^{2}$, MJO Wakelam ${ }^{1,4}$ and T Ismail ${ }^{2}$ \\ 'Cancer Research UK Institute for Cancer Studies, University of Birmingham, Birmingham B 15 2TT, UK; ${ }^{2}$ University Hospital Birmingham NHS Trust, \\ Edgbaston, Birmingham B 5 2TH, UK; ${ }^{3}$ Department of Primary Care and General Practice, University of Birmingham, Birmingham BI5 2TT, UK
}

\begin{abstract}
Early detection of polyps or colorectal carcinoma can reduce colorectal carcinoma-associated deaths. Previous studies have demonstrated raised serum levels of matrix metalloproteinase 9 (sMMP-9) in a range of cancers. The aim of this study was to investigate the role of sMMP-9 levels in identifying colorectal neoplasia. Consenting patients donated a blood sample and were assessed by proforma-led history and physical examination. Samples were analysed for sMMP-9 concentration (enzyme-linked immuno-sorbant assay) and compared to final diagnoses. Logistic regression modelling determined independent factors associated with neoplasia. A total of 365 patients were recruited of whom 300 were analysed, including 46 normal controls. A total of 27 significant adenomas and 63 malignancies were identified. The median sMMP-9 concentration was 443ng ml ${ }^{-1}$ (IQR: 219-782; mean: 546). Patients with neoplasia had significantly elevated sMMP-9 levels $(P<0.00 \mathrm{I})$. Logistic regression modelling identified elevated $\log \left(\right.$ sMMP-9) as the most significant predictor of neoplasia $\left(\chi^{2}=38.33, P<0.001\right)$. Other significant factors were age, sex, smoking history, abdominal pain and weight loss. The model accurately predicted neoplasia in $77.3 \%$ of cases. Sensitivity and specificity were 77.9 and $77.1 \%$. sMMP-9 estimation can accurately stratify patient to low- or high-risk cohorts. Serum sampling is a potential means of avoiding unnecessary colonoscopy and reducing patient anxiety, iatrogenic morbidity and mortality, and cost.

British Journal of Cancer (2007) 97, 97I-977. doi: I 0.1038/sj.bjc.6603958 www.bjcancer.com

Published online 2 October 2007

(c) 2007 Cancer Research UK
\end{abstract}

Keywords: colorectal; matrix metalloproteinase; screening; risk

Colorectal cancer remains the second commonest malignancy in England and Wales and is the target of the newest national cancer screening programme, which was officially launched in April 2006. There are more than 30000 new cases of colorectal cancer each year in the United Kingdom and about 16000 deaths, incurring an annual expenditure of more than $£ 300$ million in surgical, adjuvant, and palliative treatment (ONS, 2005; Macafee et al, 2006). Surgical advances have not improved the 5-year survival rates in the last 20 years and though an early histopathological stage at primary surgical resection correlates with increased disease-free long-term survival, $50 \%$ of patients still present with late-stage disease (Umpleby et al, 1984). Crude 5-year survival rates are only $35 \%$ due to the late stage of presentation of most cases (ONS, 2003). Resection of lesions in the premalignant stage permits cure without risk of occult distant metastases, therefore the detection of such early tumours is vital for a successful

\footnotetext{
*Correspondence: NG Hurst, Department of Surgery, Derby City General Hospital, Uttoxeter Road, Derby DE22 3NE, UK;

E-mail: Nicholas.hurst@derbyhospitals.nhs.uk

${ }^{4}$ Current address: The Babraham Institute, Babraham Research Campus, Cambridge CB22 3AT, UK

Received 25 April 2007; revised 3 August 2007; accepted 9 August 2007; published online 2 October 2007
}

screening programme. Polyp excision is important in reducing the rate of progression to carcinoma in most individuals (Muto et al, 1975; Stryker et al, 1987). Excision of polyps and early tumours is associated with a good prognosis, whereas treatment of invasive carcinomas has a less favourable outcome (Dukes, 1932). Therefore, early detection of polyps or colorectal carcinoma before invasion into the surrounding mucosa represents the best hope of reducing colorectal carcinoma-associated deaths.

The majority of investigations currently employed in the assessment of patients with colorectal symptoms involve invasive procedures, exposure to significant doses of ionising radiation, or a combination of these. One or more techniques may be sequentially used in individual assessments, with increased risk accompanying multiple investigations.

While the risk of colonic perforation may be easily assumed by the very nature of flexible endoscopic approaches, it also accounts for morbidity in radiological procedures such as contrast radiology in which the sequelae of such perforations may be exaggerated by virtue of contrast media employed. Other risks include contrast reactions and delayed effects of ionising radiation exposure, which is cumulative and non-reversible. Clearly when there is commensurate benefit in the investigation, the excess risk may be partly compensated as in the staging investigation, which may follow a positive barium enema. Recent proposals suggest that quantification of the increased lifetime risk of developing a 
radiation-induced neoplasm should be imparted to patients prior to exposure as part of a consent process (Picano, 2004). However, it is clear that a non-invasive test, avoiding ionising radiation, would be desirable.

Non-invasive faecal occult blood testing (FOBT) relies on detection of the surrogate marker of degradation products of haemoglobin. A number of factors limit its use including the relatively poor acceptability of stool sampling and that the sought tumour must bleed - not all colorectal neoplasms bleed, or may only shed blood intermittently. Friability may be a late development arising when other symptoms are apparent. Adenomatous polyps bleed less than malignant lesions, accounting for the lower sensitivity for polyps ( $30 \%$ compared to $c a .50 \%$ for carcinomas). However data are available suggesting that use of FOBT detects colorectal lesions at an earlier stage, and that screened lesions are associated with survival benefits (Towler et al, 1998).

Analysis of serum samples for stratification of risk of colorectal neoplasia is untested in the clinical setting. The ideal target moiety is debatable and has centred on carcino embryonic antigen (CEA) and non-specific inflammatory markers such as C-reactive protein, erythrocyte sedimentation rate, and serum ferritin levels. While CEA may boast a degree of specificity for malignancy, it is also elevated in many diverse states including old age, obesity, smoking, inflammatory lung disease, liver disease, and other diseases of the digestive tract (Ruibal-Morell, 1992). Elevations in CEA levels are also reported in non-colorectal malignancies, including lung (74\%) (Vincent and Chu, 1973; Dent et al, 1978), breast (57\%), oesophageal, gastric, and pancreatic carcinomas. It is also more likely to be elevated in disseminated disease than in locally confined (and hence surgically curable) lesions (Beatty et al, 1979). Levels may decline with disease regression on treatment with chemotherapy (Bates and Longo, 1987), but up to $10 \%$ of patients with falling levels exhibit progressive disease (Kreiger et al, 1983).

Matrix metalloproteinases (MMPs) are zinc-dependent endopeptidases that catalyse the dissolution of the extracellular matrix classified according to domain structure into collagenases, gelatinases, stromelysins, membrane-type, and others (Kleiner and StetlerStevenson, 1999; Pei, 1999). The gelatinases, MMP-2 and MMP-9, have been particularly implicated in tumour invasion and metastasis formation (Poulsom et al, 1992; Parsons et al, 1998; Zeng et al, 1999). The primary substrate for these enzymes is type IV collagen, a major component of the basement membrane, which represents a substantial barrier for tumour cell metastasis. Most MMPs are secreted as zymogens and require activation through cleavage of a pro-domain sequence located at the $\mathrm{N}$ terminus of the protein.

MMPs are elevated during physiological processes, such as tissue remodelling during growth phases, pregnancy and partuition, following injury and in pathological disease states, for example chronic inflammatory processes and malignancy (BirkedalHansen et al, 1993). Their role in malignancy is highlighted by the number of cancers that have elevated MMP levels, including breast (Basset et al, 1990), prostatic (Pajouh et al, 1991), gastric (Nomura et al, 1995), ovarian (Campo et al, 1992), pancreatic (Gress et al, 1995; Bramhall et al, 1997), and colorectal disease (Hewitt et al, 1991; Gallegos et al, 1995). This led to great interest in development of MMP inhibitors as potential tumour-control agents. Unfortunately, clinical trials have failed to demonstrate successful curtailment of tumour progression (Greenwald, 1999; Steward, 1999). Nevertheless their elevation in a range of cancers prompted us to address the possibility that their levels may be elevated in the serum of patients with colorectal cancer.

\section{AIMS}

The aims of this study were to (1) investigate the prophetic role of elevated serum matrix metalloproteinase 9 (sMMP-9) levels in identifying colorectal neoplasia within a symptomatic cohort and (2) assign and quantify relative risk of neoplasia based on serum analysis, in order to prioritise for, or even exclude from, further investigation.

\section{MATERIALS AND METHODS}

Ethical approval was provided by South Birmingham REC and University Hospital Birmingham NHS Trust R\&D office (857/04; RRK2544). All adult patients referred to a specialist colorectal clinic over a 12-month period were invited to participate. After giving informed consent, study subjects donated a sample of blood, which was encoded. All patients then were clinically assessed by proforma-led history and physical examination including rigid sigmoidoscopy. Protocol-driven invasive investigation ensued and patients were tracked to a definitive diagnosis. Serum samples were analysed for MMP-9 concentration and compared to decoded patient diagnoses. Diagnostic grouping were coined to facilitate analysis (Table 1) and a hierarchy of diagnoses was employed (data not shown) to express in simple terms the most significant pathology identified.

Patients unable or unwilling to give informed consent were excluded and referred for investigation as per clinic protocols. Patients who did not attend for subsequent investigation were excluded from the study if they did not respond to reminders generated by the imaging department (independent of the study).

Table I Diagnostic coding

\begin{tabular}{|c|c|}
\hline & Coding \\
\hline \multicolumn{2}{|l|}{ Diagnosis } \\
\hline Normal control & I \\
\hline No abnormality detected on investigation (NAD) & 2 \\
\hline Haemorrhoids & 3 \\
\hline Irritable bowel syndrome & 4 \\
\hline Diverticular disease & 5 \\
\hline Inflammatory bowel disease & 6 \\
\hline Other anal/colorectal non-neoplastic pathology & 7 \\
\hline Other non-colorectal non-neoplastic pathology & 8 \\
\hline Non-premalignant polyp (metaplastic) & 9 \\
\hline Adenomatous polyp & 10 \\
\hline Colorectal carcinoma & | | \\
\hline Non-colorectal malignancy (primary diagnosis) & 12 \\
\hline Other 'screenworthy' pathology (non-neoplastic) & 13 \\
\hline \multicolumn{2}{|l|}{ Diagnostic grouping } \\
\hline Normal/no screenworthy pathology & $1,2,3,4,5,6,7,8,9$ \\
\hline Normal/no detectable disease & 1,2 \\
\hline Benign non-screenworthy pathology & $2,3,4,5,6,7,8,9$ \\
\hline Colorectal adenomatous polyp & 10 \\
\hline Colorectal malignancy & | | \\
\hline Colorectal malignant and premalignant & 10,11 \\
\hline Screenworthy pathology & $10,11,12,13$ \\
\hline
\end{tabular}

Normal control: laboratory disease-free volunteers. NAD: patients without positive findings on colorectal imaging, and includes patients with self-limiting symptoms as well as asymptomatic individuals requesting screening investigation for poor family histories in whom no lesion was identified. Diverticular disease: patients with proven diverticulosis and symptoms compatible with the clinical manifestations of the condition, without evidence of septic complications. Inflammatory bowel disease includes Crohn's disease, ulcerative colitis, and non-specific colitis. Other anal/ colorectal non-neoplastic pathology includes conditions such as fissure and nonspecific pruritis ani. Other non-colorectal non-neoplastic pathology includes diverse conditions such as coeliac disease, ovarian cystic disease, and vascular conditions. Metaplastic polyps were considered to have no malignant potential, while adenomatous polyps were considered potentially pre-malignant entities. Noncolorectal malignancies included those of ovarian, gastric, haematological, and renal origin. Screenworthy pathology (code ' 13 ') includes diverticular strictures requiring surgical intervention for diagnostic discrimination from malignancy. 


\section{Serum preparation}

Blood was harvested by single venepuncture, into plain tubes using the proprietary Vacutainer ${ }^{B}$ system, prior to eliciting histories or physical examination. After clotting, serum was separated (centrifuged at $2000 \mathrm{~g}$ ) and aliquoted to polyethylene Eppendorf vials for storage at $-80^{\circ} \mathrm{C}$ until analysis.

\section{MMP-9 ELISA}

MMP-9 ELISA kits (R\&D Systems, UK) were used according to the manufacturer's instructions. Serum samples were diluted 100-fold in assay buffer. All assays were performed in duplicate on each of two enzyme-linked immuno-sorbant assay (ELISA) plates assayed 2 weeks apart, and concordance within $10 \%$ for all four wells was required. Serum MMP-9 (sMMP-9) concentrations from 'diseasefree normal control' samples declined with advancing age ( $n=46$; $\left.R^{2}=0.027\right)$ and, therefore, age-specific predicted (expected) sMMP-9 were generated. Ratios of observed/expected values were calculated; a ratio of less or equal to 1 was considered normal, while ratios above 1 were considered pathologically elevated. A sample was denoted 'positive' if the observed to expected ratio exceeded 1, and a similar prospective prediction of the presence of underlying colorectal neoplasia was declared, prior to breaking the identifying sample code. The underlying definitive diagnosis was compared to the prospective prediction and accuracy recorded. Total sMMP-9 concentrations were also recorded and mapped to diagnostic group.

\section{Statistical analyses}

To identify associations in the data, Pearson's $\chi^{2}$ test was used for categorical variables. The non-parametric Wilcoxon two-sample test (W) and Kruskal - Wallis test (KW) for more than two samples were used to compare groups of continuous measurements. Odds ratios (OR) were calculated to determine the association between exposure to possible risk factors and outcome. ORs, adjusted for all other predictive variables, were derived from logistic regression analyses. Non-neoplastic diagnoses were combined (nonneoplastic group), as were pre-malignant adenomas and colorectal carcinomas ((pre-) malignant group) in order to devise a logistic regression model to 'predict' patients with (pre-) malignant disease. All available factors were considered in the logistic regression analyses (gender, age, sMMP-9 level ( $\mathrm{ng} \mathrm{ml}^{-1}$ ) under a log transformation, family history, rectal bleeding, altered bowel habit, abdominal pain, weight loss, and smoking history). Logistic regression analyses using backward elimination of variables were performed using a $5 \%$ significance level to determine independent factors for prediction of those with pre-malignant or established malignant disease. Model accuracy was calculated and sensitivity, specificity, proportion of false positives, and negatives and overall percentage of correct predictions were presented. The probability cut-point was chosen to balance sensitivity and specificity.

\section{RESULTS}

\section{Serum MMP-9 concentration analysis}

A total of 365 patients were seen by a single clinician in the QED (Quick and Early Diagnosis) specialist colorectal clinic over a 12month period. Onward referral for further investigation occurred in 332 cases. Informed and consenting patients donated serum for analysis. Eighteen patients declined to participate and 14 patients did not attend for their investigations. Three hundred patients with a definitive diagnosis (achieved largely by double-contrast enema) and matched serum samples were therefore enrolled in the serological arm of this study. These comprised 134 males (median (IQR) age: $62(52-71)$ years) and 166 females (median (IQR) age:
$64(51-76)$ years). Twenty-seven significant adenomas (multiple $>3$, severe dysplasia, $>1 \mathrm{~cm}$ diameter with predominant villous component) and 63 malignancies were identified in the study population. All Duke's stages were represented in the population (Stage A: 13 (20.9); B; 21 (33.9); C: 20 (32.3); D: 9 (12.9\%)). Fortysix normal controls (healthy laboratory and hospital staff including retired voluntary workers) were recruited and bled (18 males, median age 52 years (inter-quartile range 46-65); 28 females, median age 62 years (inter-quartile range 51-73)).

\section{Observed to predicted ratios}

There was a wide variation in ELISA-determined sMMP-9 concentration. Nevertheless a median sMMP-9 control value of $261 \mathrm{ng} \mathrm{ml}^{-1}$ was obtained (IQR: $105-520$; mean $=340$ ) in these 46 patients. Sample repeat analysis after storage (at $-80^{\circ} \mathrm{C}$ ) did not affect detected MMP9 concentrations. Several non-neoplastic conditions were diagnosed in the sample group including haemorrhoids, diverticular disease, irritable bowel syndrome, and colitis (Table 2). In each of these individual conditions the mean SMMP-9 concentration was not significantly greater than the control value. In contrast, patients with pre-malignant adenomas or colorectal carcinoma had significantly elevated sMMP-9 levels.

Ratios of observed to predicted values of sMMP-9 concentration, corrected for patient age, demonstrated the ability to differentiate between non-neoplastic and neoplastic disease (Figure 1). Significant differences were demonstrable between sMMP9

Table 2 Non-neoplastic conditions represented in symptomatic patient group

\begin{tabular}{lrr}
\hline Diagnostic category & $\boldsymbol{n}$ & $\%$ \\
\hline NAD & 46 & 23 \\
Haemorrhoids & 25 & 12 \\
Diverticular disease & 85 & 42 \\
Irritable bowel syndrome & 7 & 3 \\
Inflammatory bowel disease & 14 & 7 \\
Other colorectal non-neoplastic & 13 & 6 \\
Non-colorectal non-neoplastic & 10 & 5 \\
Metaplastic polyp & 5 & 2 \\
& & 100 \\
Total & 205 & \\
\hline
\end{tabular}

Abbreviation: NAD = no abnormality detected.

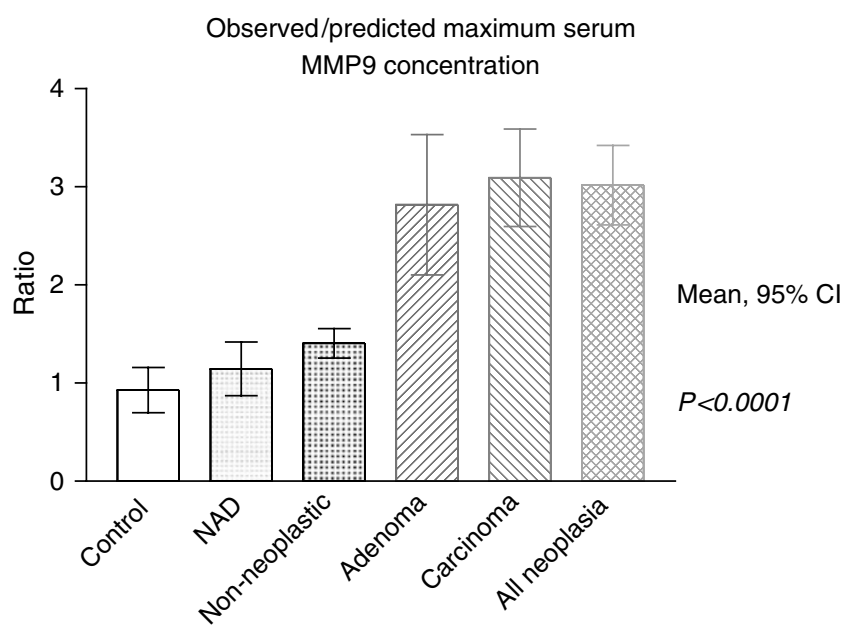

Figure I Observed to predicted maximum serum matrix metalloproteinase 9 (sMMP-9) concentrations. Highly significant differences between non-neoplastic and neoplastic conditions demonstrated. 
concentrations in non-neoplastic and neoplastic conditions $(P<0.0001)$. No statistically significant differences were identified between controls and no abnormality detected (NAD) groups $(P=0.2794)$, or between NAD groups and non-neoplastic patients $(P=0.1490)$.

A patient-specific prediction of the presence or absence of screenworthy pathology (see Table 1) was made based on the observed to predicted ratio of sMMP-9. This was compared to definitive diagnoses to ascertain accuracy of prediction, results of which can be seen in Table 3. The overall accuracy of this prediction was $61.0 \%$, with a false negative rate of $1.3 \%$; one lesion was visible in the mid-rectum giving an effective clinical falsenegative rate of $1 \%$. However, this was at the expense of an increased false-positive rate of $37.7 \%$. Positive and negative predictive values were 44.6 and $95.8 \%$, respectively. It is particularly noteworthy that no pre-malignant lesions were overlooked by this method. The raw cancer rate in our referred study population was $22.7 \%$, rising to $31.4 \%$ in those with a positive serological ratio; similarly the pre-malignant adenoma rate rose from 9 to $13.2 \%$ under the same conditions. Overall (pre)malignant lesion rates rose from 31.7 to $44.6 \%$ (a $40.7 \%$ increase). Restriction of invasive investigations to only those with positive serology could reduce imaging requirements by $32 \%$, risking overlooking four lesions, of which one was visible in the midrectum, or $4.2 \%$ of screenworthy pathology. Alternatively, prioritising the patients for investigation on the basis of serological results may reduce time to detection in those with positive invasive investigations.

\section{Absolute serum MMP-9 concentrations}

Progressing from individual patient predictions, an assessment was made of the probability of specific levels of sMMP-9, in groups with particular characteristics, indicating the presence of a screenworthy lesion. Additionally the contribution of each of several variant factors to the risk of harbouring colorectal (pre)malignant disease was calculated.

Measured sMMP-9 concentrations were recorded against multiple factors postulated potentially relevant to modification of risk of colorectal neoplasia. Subjects were categorised as symptomatic non-neoplastic $(n=205)$ including 46 normal controls, or colorectal (pre)-malignant $(n=95)$. Factors considered in logistic regression analysis included sMMP-9 level (under a log transformation), age, gender, smoking history (never $v s$ ever), family history (negative $v s$ positive), rectal bleeding (negative $v s$ positive), persistent altered bowel habit (negative $v s$ positive), unexplained abdominal pain (negative $v s$ positive), and unexplained weight loss (negative vs positive). The median sMMP-9 levels between the various non-neoplastic and control categories (see Table 1) did not significantly differ (KW $\chi^{2}=5.69, P=0.58$ ) and groups were combined as the non- (pre)-malignant group $(n=205)$. sMMP-9 concentrations in the pre-malignant group $(n=27)$ and the colorectal malignant group $(n=59)$ were statistically similar

Table 3 Comparison of prediction based on observed to predicted serum MMP-9 ratios to presence or absence of screenworthy pathology

\begin{tabular}{lccr}
\hline & \multicolumn{2}{c}{ Observed/predicted ratio } \\
\cline { 2 - 3 } & +ve $(>\mathbf{I . 0})$ & $-\mathbf{v e}(\leqslant \mathbf{I . 0})$ & \\
\hline SWP & & & \\
Yes & 91 & 4 & 205 \\
No & 113 & 92 & 300 \\
& 204 & 96 & \\
\hline
\end{tabular}

Abbreviation: $\mathrm{SWP}=$ screenworthy pathology. Sensitivity: $44.6 \%$, specificity: $95.8 \%$ positive predictive value: $95.8 \%$, negative predictive value: $44.9 \%$.
(KW $\chi^{2}=3.38, P=0.34$ ), confirming suitability for their combination as a single group.

The risk of neoplastic disease at univariate analysis was significantly associated with increased age, male gender, increased sMMP-9, and paucity of index symptoms, other than weight loss (Table 4). Logistic regression analysis sought to identify the contribution of each variable to the risk of neoplastic disease multivariably utilising the complete data set of 300 patients in the final logistic regression model, including 95 patients with malignant or pre-malignant pathology. The final model identified sMMP-9 as the single most significant predictor of (pre)-malignant disease $\left(\chi^{2}=38.33, P<0.001\right)$. Other significant independent predictors of neoplastic disease identified were advancing age $\left(\chi^{2}=11.25, \quad P<0.001\right)$, male gender $\left(\chi^{2}=8.73, P=0.003\right)$, a negative smoking history $\left(\chi^{2}=4.87, P=0.027\right)$, absence of abdominal pain $\left(\chi^{2}=4.72, P=0.030\right)$, and increased weight loss $\left(\chi^{2}=4.70, P=0.030\right)$. The other three variables (rectal bleeding, altered bowel habit, or family history) did not achieve statistical significance for inclusion, in keeping with the findings of the barium enema study previously described.

Furthermore, the risk of being in the (pre)-malignant group, attributable to a 1-unit increase in $\log ($ sMMP-9) concentration is estimated as a 5.09-fold risk (95\% confidence interval (CI): 3.04 , 8.51). The difference in median sMMP-9 concentrations between non-neoplastic and (pre)-malignant groups was $443 \mathrm{ng} \mathrm{ml}^{-1}$. Similar calculations are possible for aging patients, who demonstrate a $4 \%$ increased risk per additional year (95\% CI: $2-7 \%$ ) generating a risk elevation of $66 \%$ per advancing decade of life. The model estimated increased risks of neoplastic disease for men $(\mathrm{OR}=2.52$; $95 \% \mathrm{CI}: 1.37,4.66)$, non-smokers $(\mathrm{OR}=0.48 ; 95 \% \mathrm{CI}$ : $0.25,0.92)$, absence of abdominal pain $(\mathrm{OR}=0.49 ; 95 \% \mathrm{CI}: 0.26$, $0.93)$, and weight loss $(\mathrm{OR}=2.60 ; 95 \% \mathrm{CI}$ : $1.10,6.15)$ (Table 5).

A probability equation can be derived from data held in Table 5 to enable calculation of the probability of an individual patient, with defined characteristics, being included in the (pre)-malignant group (Figure 2).

Moderation of the probability threshold denoting inclusion in the (pre)-malignant group defines the accuracy of the prediction generated.

If a probability threshold to balance sensitivity and specificity is assumed, the model accurately predicts inclusion or exclusion from this group in $77.3 \%$ of patients, for this sample. Sensitivity and specificity are 77.9 and $77.1 \%$, respectively. However, falsely negative predictions are produced in 21 individuals $(11.7 \%$ of negative predictions, $7.0 \%$ of all predictions). Falsely positive predictions are derived in 47 patients $(38.8 \%$ of positive predictions, $15.7 \%$ of all predictions).

Patient groups may be defined based on age, gender, smoking history, abdominal pain, weight loss, and ultimately sMMP-9 concentration to provide estimated risks of colorectal neoplasia.

In the best case scenario, a 32-year old, female smoker with abdominal pain, no weight loss, and an sMMP-9 concentration of 263 , would have a calculated probability of colorectal neoplasia of $1.1 \%$. The same woman with an sMMP-9 concentration of $1025 \mathrm{ng} \mathrm{ml}^{-1}$ would have an eightfold elevation of her risk $(8.9 \%)$. In the worst case scenario, a 79-year old, male, nonsmoker with no abdominal pain, increased weight loss and an sMMP-9 level of 895 , would have a calculated risk of colorectal neoplasia of $93.5 \%$.

\section{CONCLUSIONS AND DISCUSSION}

Serum MMP-9 estimation may facilitate stratifying patients referred to a specialist rapid access colorectal cancer unit into low- or high-risk cohorts, when analysed by ELISA. If our results are validated in further studies, individualised prediction of the risk of colorectal neoplasia may be inferred by comparison to local 


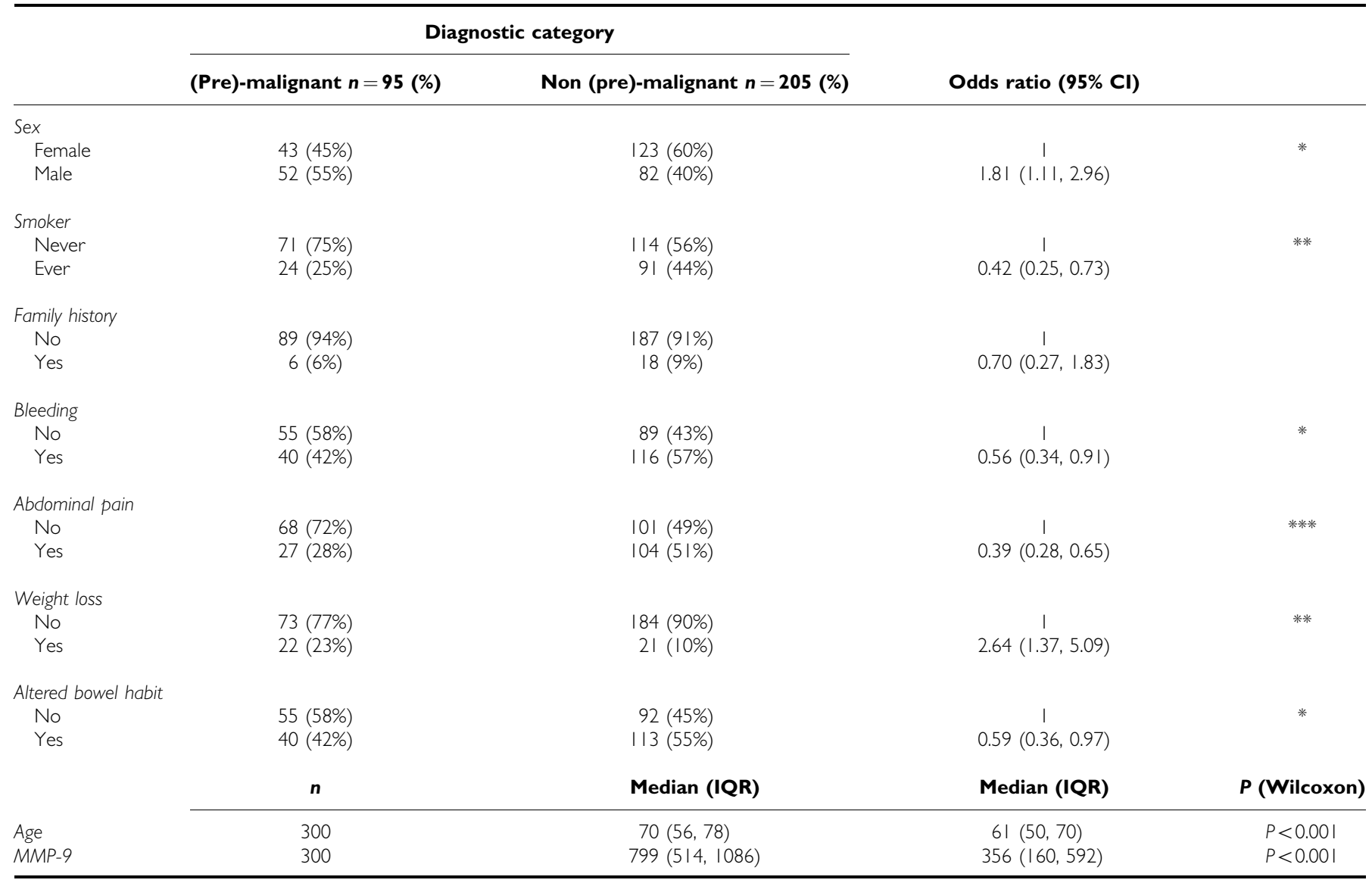

Abbreviations: $\mathrm{Cl}=$ confidence interval; MMP-9 $=$ metalloproteinase 9. $* * * P<0.00$ I, $* * P<0.0$ I, $* P<0.05$ when using $\chi^{2}$ analysis. Odds ratios are given for likelihood of (pre)malignant disease, for any given univariate discriminator.

Table 5 Logistic regression model for the prediction of (pre)-malignant disease

\begin{tabular}{llrccc}
\hline Variable & Calculation & $\boldsymbol{\beta}$ & $\boldsymbol{\chi}^{\mathbf{2}}$ & $\boldsymbol{P}$ & Odds ratio (95\% CI) \\
\hline Intercept & - & -13.427 & 44.03 & $<0.001$ & - \\
LogMMP-9 & Continuous & 1.626 & 38.33 & $<0.001$ & $5.09(3.04,8.51)$ \\
Age & Continuous & 0.040 & 11.25 & $<0.001$ & $1.04(1.02,1.07)$ \\
Sex & Female vs male & 0.925 & 8.73 & 0.003 & $2.52(1.37,4.66)$ \\
Smoking history Never vs ever & -0.731 & 4.87 & 0.027 & $0.48(0.25,0.92)$ \\
Abdominal pain No vs yes & -0.718 & 4.72 & 0.030 & $0.49(0.26,0.93)$ \\
Weight loss & No vs yes & 0.954 & 4.70 & 0.030 & $2.60(1.10,6.15)$ \\
\hline
\end{tabular}

Abbreviation: $\mathrm{Cl}=$ confidence interval. Adjusted $R^{2}=0.44$.

Probability of inclusion in (pre)-malignant diagnostic category
$Z=-13.427+1.626($ log MMP-9) +0.040 (age) +0.925 (sex) -0.731 (smoking history) -
0.718 (abdominal pain) +0.954 (weight loss)
$P=\frac{\exp (Z)}{1+\exp (Z)}$
Sex: $0=$ female, $1=$ male
Smoking history: $0=$ never, $1=$ ever
Abdominal pain: $0=$ no, $1=$ yes
Weight loss: $0=$ no, $1=$ yes

Figure 2 Logistic regression equations for calculation of individual risk. population profiles of sMMP-9 concentrations, with accuracy in excess of $76 \%$, far exceeding that obtainable by the primary noninvasive stratification tool in current use in the United Kingdom (guaiac FOBT). While more specific methods of faecal occult blood detection are available (including immunological iFOBT, eg, Hemeselect ${ }^{\mathbb{R}}$ and Quickview $\left.{ }^{\mathbb{R}}\right)$ their increased cost has, to date, precluded use in the bowel screening programme. Heightened sensitivity may be obtained by faecal DNA analysis (PreGenPlus ${ }^{\circledR}$ ) which improves detection of colorectal neoplasia from 12.9 to $51.6 \%$ compared to standard guaiac-based FOBT (Imperiale et al, 2004), but these techniques are expensive ( $\$ 575 /$ test at commercial rates) and their use is, at present, limited to the research setting.

Quantification of the probability of neoplasia is modifiable according to local preference. If exclusion of falsely negative test results is desirable in order to reduce the total burden of invasive imaging, sensitivity may be as high as $96.4 \%$ (rising to $99.7 \%$ in combination with clinical assessment), by altering the probability threshold calculations to favour sensitivity. If conversely, simple stratification is desirable to prioritise imaging appointments, modifying the probability threshold to 0.3 increases the positive imaging rate from 29 to $57 \%$.

Secondary 'sieving' of patients with positive FOBTs returned in the screening round of the national colorectal cancer screening programme could be performed by means of ELISA quantification of sMMP-9. Negative serum samples could be excluded from confirmatory colonoscopy, significantly reducing the burden on this service. The cost savings generated could amount to a sizeable proportion of the running costs of the national FOBT screening 
programme. Furthermore, avoidance of unnecessary colonoscopy has the added benefit of reducing patient anxiety and potential for iatrogenic morbidity and mortality, with associated secondary cost savings accrued.

Limitations of this method of risk stratification include the introduction of human error into the assay protocol, resulting in inaccurate ELISA data with potentially damaging results. However, duplication and quality control concordance checks could help to minimise this risk and automation would reduce technical variations between assay plates.

This study was undertaken in a selected population with a high prevalence of malignancy; the usefulness of sMMP9 as a primary or secondary screening test may vary in population with different prevalence of disease. Nevertheless, this study suggests that sMMP9 may have potential application: (1) normal sMMP9 levels may be a means of identifying those at low risk of malignant disease and avoiding the need for invasive investigations and (2) raised sMMP9 levels may be a means of identifying those at increased risk of malignancy. Further research in larger, more representative populations is required to determine the usefulness of sMMP9 in stratifying both symptomatic and asymptomatic

\section{REFERENCES}

Basset P, Bellocq JP, Wolf C, Stoll I, Hutin P, Limacher JM, Podhajcer OL, Chenard MP, Rio MC, Chambon P (1990) A novel metalloproteinase gene specifically expressed in stromal cells of breast carcinomas. Nature 348: $699-704$

Bates SE, Longo DL (1987) Use of serum markers in cancer diagnosis and management. Semin Oncol 14: 102-138

Beatty JD, Romero C, Brown PW, Lawrence Jr W, Terz JJ (1979) Clinical value of carcinoembryonic antigen: diagnosis, prognosis, and follow up of patients with cancer. Arch Surg 114: $563-567$

Birkedal-Hansen H, Moore WG, Bodden MK, Windsor LJ, Birkedal-Hansen B, DeCarlo A, Engler JA (1993) Matrix metalloproteinases: a review. Crit Rev Oral Biol Med 4: 197-250

Bramhall SR, Neoptolemos JP, Stamp GW, Lemoine NR (1997) Imbalance of expression of matrix metalloproteinases (MMPs) and tissue inhibitors of the matrix metalloproteinases (TIMPs) in human pancreatic carcinoma. J Pathol 182: 347-355

Campo E, Merino MJ, Tavassoli FA, Charonis AS, Stetler-Stevenson WG, Liotta LA (1992) Evaluation of basement membrane components and the $72 \mathrm{kDa}$ type IV collagenase in serous tumors of the ovary. Am J Surg Path 16: $500-507$

Dent PB, McCulloch PB, Wesley-James O, MacLaren R, Muirhead W, Dunnett CW (1978) Measurement of carcinoembryonic antigen in patients with bronchogenic carcinoma. Cancer 42: 1484-1491

Dukes CE (1932) The classification of cancer of the rectum. J Pathol Bacteriol 35: 323-332

Gallegos NC, Smales C, Savage FJ, Hembry RM, Boulos PB (1995) The distribution of matrix metalloproteinases and tissue inhibitor of metalloproteinases in colorectal cancer. Surg Oncol 4: 111-119

Greenwald RA (1999) Thirty-six years in the clinic without an MMP inhibitor. What hath collagenase wrought? Ann NY Acad Sci 878: $413-419$

Gress TM, Muller-Pillasch F, Lerch MM, Friess H, Buchler M, Adler G (1995) Expression and in-situ localization of genes coding for extracellular matrix proteins and extracellular matrix degrading proteases in pancreatic cancer. Int J Cancer 62: 407-413

Hewitt RE, Leach IH, Powe DG, Clark IM, Cawston TE, Turner DR (1991) Distribution of collagenase and tissue inhibitor of metalloproteinases (TIMP) in colorectal tumours. Int J Cancer 49: 666-672

Imperiale TF, Ransohoff DF, Itzkowitz SH, Turnbull BA, Ross ME, Colorectal Cancer Study Group (2004) Fecal DNA vs fecal occult blood for colorectal cancer screening in an average-risk population. $N$ Engl J Med 351: 2704-2714

Kleiner DE, Stetler-Stevenson WG (1999) Matrix metalloproteinases and metastasis. Cancer Chemother Pharmacol 43: S42-S51

Kreiger G, Wander HE, Prangen M, Bandlow G, Beyer JH, Nagel GA (1983) Determination of the carcinoembryonic antigen (CEA) for predicting the populations and determining any regional or ethnic variations in normal sMMP-9 profiles. This preliminary work has informed further ongoing work aiming to evaluate the role of sMMP9 in detecting (pre)-malignant disease in more typical populations (Ryan et al, 2006; Wilson et al, 2006).

The detection of colorectal neoplasia by this method is not infallible, but this work demonstrates that it has the potential to add to existing methodologies. It identifies that the capability exists not only for detection of established malignancy, but also for pre-malignant lesions with the same accuracy as their invasive counterparts. No other methods currently in the public domain have shown the ability to identify colorectal adenomas by noninvasive means with this degree of accuracy.

\section{ACKNOWLEDGEMENTS}

This work was supported by grants from Cancer Research UK and the Medical Research Council to Michael Wakelam. Sue Wilson is funded by a Department of Health Career Scientist Award. success of therapy in metastatic breast cancer. Dtsch Med Wochenschr 108: $610-614$

Macafee DAL, Gemmill EH, Lund JN (2006) Colorectal cancer: current care, future innovations and economic considerations. Expert Rev Pharmacoeconomics Outcomes Res 6: 195-206

Muto T, Bussey HJR, Morson BC (1975) The evolution of cancer of the colon and rectum. Cancer 36: 2251-2270

Nomura H, Sato H, Seiki M, Mai M, Okada Y (1995) Expression of membrane-type matrix metalloproteinase in human gastric carcinomas. Cancer Res 55: 3263-3266

Office for National Statistics (2003) Cancer survival, England and Wales, 1991-2001, http://www.statistics.gov.uk/statbase/ssdataset.asp?vlnk = 7091

Office for National Statistics (2005) Cancer Statistics Registrations: Registrations of Cancer Diagnosed in 2002, England. Series MB1 33. London: National Statistics

Pajouh MS, Nagle RB, Breathnach R, Finch JS, Brawer MK, Bowden GT (1991) Expression of metalloproteinase genes in human prostate cancer. J Cancer Res Clin Oncol 117: 144-150

Parsons SL, Watson SA, Collins HM, Griffin NR, Clarke PA, Steele RJ (1998) Gelatinase (MMP-2 and -9) expression in gastrointestinal malignancy. $\mathrm{Br}$ J Cancer 78: 1495 - 1502

Pei D (1999) Identification and characterization of the fifth membranetype matrix metalloproteinase MT5-MMP. J Biol Chem 274: 89258932

Picano E (2004) Informed consent and communication of risk from radiological and nuclear medicine examinations: how to escape from a communication inferno. BMJ 329: 849-851

Poulsom R, Pignatelli M, Stetler-Stevenson WG, Liotta LA, Wright PA, Jeffrey RE, Longcroft JM, Rogers L, Stamp GW (1992) Stromal expression of $72 \mathrm{kDa}$ type IV collagenase (MMP-2) and TIMP-2 mRNAs in colorectal neoplasia. Am J Pathol 141: 389-396

Ruibal-Morell A (1992) CEA serum levels in non-neoplastic disease. Int $J$ Biol Markers 7: $160-166$

Ryan A, Wilson S, Wakelam M, Warmington S, Dunn J, Hobbs R, Martin A, Ismail T 2006 A prospective study to assess the value of MMP-9 in improving the appropriateness of urgent referrals for colorectal cancer. BMC Cancer 6: 251

Steward WP (1999) Marimastat (BB2516): current status of development. Cancer Chemother Pharmacol 43: S56-S60

Stryker SJ, Wolff BG, Culp CE, Libbe SD, Ilstrup DM, MacCarty RL (1987) Natural history of untreated colonic polyps. Gastroenterology 93: $1009-1013$

Towler B, Irwig L, Glasziou P, Kewenter J, Weller D, Silagy C (1998) A systemic review of the effects of screening for colorectal cancer using the faecal occult blood test, hemoccult. BMJ 317: 559-565 
Umpleby HC, Bristol JB, Rainey JB, Williamson RC (1984) Survival of 727 patients with single carcinoma of the large bowel. Dis Colon Rectum 27: 803-810

Vincent RG, Chu TM (1973) Carcinoembryonic antigen in patients with carcinoma of the lung. J Thorac Cardiovasc Surg 66: 320 - 328

Wilson S, Wakelam MJO, Hobbs FDR, Ryan AV, Dunn JA, Redman VD, Patrick F, Colbourne L, Martin A, Ismail T 2006 Evaluation of the accuracy of serum MMP-9 as a test for colorectal cancer in a primary care population. BMC Cancer 6: 258

Zeng ZS, Cohen AM, Guillem JG (1999) Loss of basement membrane type IV collagen is associated with increased expression of metalloproteinases 2 and 9 (MMP-2 and MMP-9) during human colorectal tumorigenesis. Carcinogenesis 20: $749-755$ 